\title{
¿Están los futuros profesores formados en inclusión?: Validación de un cues- tionario de evaluación ${ }^{1}$
}

\author{
Francisca González-Gil, Elena Martín-Pastor, Begoña Orgaz Baz \\ Universidad de Salamanca. España
}

\section{RESUMEN}

El objetivo de este trabajo es presentar la validación de una escala para evaluar la formación inicial en educación inclusiva de estudiantes universitarios de las titulaciones de educación. Para ello se partió de una escala que ha pasado ya una primera validación dirigida a profesionales en ejercicio (CEFI-R). Se contó con una muestra de 202 profesores españoles que impartían docencia en todas las etapas educativas y con 495 estudiantes universitarios de los grados de maestro y pedagogía y del máster de secundaria. Se describe el desarrollo del proceso de validación, que ha dado como resultado un cuestionario con 16 ítems agrupados en 4 dimensiones de inclusión: concepción de la diversidad, metodología, apoyos y participación de la comunidad.

Palabras Clave: formación inicial, cuestionario, validación, educación inclusiva, estudiantes universitarios.

\section{¿Are future teachers trained for inclusion?: Validation of an assessment questionarie}

\section{ABSTRACT}

The aim of this research is the review and validation of an instrument to assess future teachers training needs for inclusion. To do this, it was used a validated instrument for inservice teachers (CEFI-R). For this purpose, we used a sample of 202 Spanish teachers with experience in all educational stages and 495 students from education Grades and Posgrades. This work describes the validation process, which resulted in the development of a 16-item questionnaire grouped into 4 inclusion indicators: conception of diversity, methodology, supports, and community participation.

Keywords: initial teacher training, questionnaire, validation, inclusive education, university students.

\begin{abstract}
"Vivimos tiempo turbulentos. El mundo está rejuveneciendo y aumentan las aspiraciones a los derechos humanos y a la dignidad

(...). El mundo está cambiando: la educación debe cambiar también"
\end{abstract}

(UNESCO, 2015, p. 3)

\section{Introducción}

Son numerosas las investigaciones que enfatizan los beneficios de la inclusión educativa-democrática, justa y equitativa- (Dias y Cadime, 2016; Donelly y Watkins, 2011; Escudero y Martínez, 2011; Robinson, 2017; Sharma y Jacobs, 2016). Una educación que da la bienvenida a todos los alumnos, no a un extraño y que se adapta para que todo el que tiene derecho a estar, esté; una educación que, en definitiva, no tendría que ir acompañada de la palabra inclusión (Pujolás, 2015; Skliar, 2013). Aun así, se trata de un término que se ha integrado en la cotidianeidad de los discursos políticos, de la legislación, de la literatura peda- gógica, de los planes de estudio universitarios y de las escuelas (Arnáiz y Guirao, 2015). La propia UNESCO (2017) destaca como uno de los principales retos de la agenda 2030 la transformación de la educación para que ésta sea abierta a todos, sea inclusiva y de buena calidad. Sin embargo, mientras que los discursos a favor de una educación inclusiva han evolucionado hasta llegar a generalizarse, las prácticas educativas que se desarrollan diariamente en las aulas no han corrido la misma suerte.

En este sentido, ha quedado ampliamente constatado (Avramidis y Kalyra, 2007; Colmenero, Pantoja y Pegajalar, 2015; Forteza, 2011; Kraska y Boyle, 2014; Loreman, et al., 2016; Molina y Holland, 2010; Rodríguez-Gómez, Armengol y Meneses, 2017; Sharma y Jacobs, 2016) cómo el éxito de la educación y, por ende, de la inclusión, depende en gran medida de la preparación del profesorado, de sus competencias y actitudes para enseñar en un entorno donde la diversidad es un aspecto que suma en el proceso de enseñanza-aprendizaje de los alumnos. Sin embargo, auto-

Trabajo financiado por el Vicerrectorado de Investigación y Transferencia de la Universidad de Salamanca

Recibido: 15.02.2017

Aceptado: 06.03.2017

ISSN: 0210-2773

DOI: https://doi.org/10.17811/rifie.46.2017.33-40 
res como Molina y Holland (2010) señalan que es precisamente la falta de formación de los docentes una de las principales dificultades en el tema que nos ocupa. De hecho trabajos previos (Acedo, 2011, Colmenero et al., 2015) advierten de la poca atención que se ha venido prestando a la formación de profesores competentes, atentos y sensibles a la diversidad de realidades presentes en el aula. Una formación que continúa infranqueable y que, en muchas ocasiones, se resume en una lista simplista de consejos prácticos y estrategias o recursos didácticos divididos por la categorización impuesta de alumnos en función de sus necesidades, dificultades o características. Lo anterior en contraposición de un planteamiento formativo que convierta a los futuros docentes en personas que hagan elecciones reflexivas respaldadas por un sólido conocimiento profesional, a fin de brindar igualdad de oportunidades y altas expectativas de éxito para todos los alumnos (Florian, Young y Rouse, 2010; Loraine, 2015; Muntaner, Roselló y de la Iglesia, 2016; Spratta y Florian, 2015). Y todo ello en el mejor de los casos, ya que en otros estudios internacionales citados por Reoyo, Carbonero y Martín (2017) el panorama es más desolador al presentarse listados con indicadores de los rasgos que definen a un maestro eficaz, en los cuales no aparece ninguna mención expresa a la formación o a la capacidad para dar respuesta a las necesidades de sus alumnos desde la óptica de la inclusión.

La UNESCO en sus documentos más recientes (2015, 2016, 2017) incide en lo fundamental y necesaria que es la inversión en la mejora de la formación y cualificación del profesorado (especialmente en inclusión) y en cómo la enseñanza superior, como contexto de formación de la cantera docente, es la piedra angular del desarrollo sostenible al tener que dotar de conocimientos y competencias específicas y promover valores básicos como libertad, tolerancia y dignidad.

Así aparece recogido también en la definición de la misión de la Universidad (Bricall, 2000) donde, entre otras cosas, se incide en la formación de profesionales motivados, con inquietudes para seguir conociendo y para enfrentarse de forma autónoma a los cambios y nuevos retos de la sociedad en general y del sistema educativo en particular y destacado por Muntaner, Rosselló y De la Iglesia (2016, p. 46): “aprender a trabajar en los centros escolares con la diversidad del alumnado, manteniendo la igualdad de oportunidades y las altas expectativas de éxito para todos los alumnos, representa los dos grandes retos del modelo educativo actual".

De esta forma, en el contexto de las titulaciones universitarias vinculadas con el ámbito educativo se asumen, además de las competencias transversales instrumentales, interpersonales y sistémicas necesarias para todo profesional competitivo y de calidad, una serie de competencias que deben estar presentes, de una manera u otra, en las titulaciones de educación (ANECA, 2005). Se destacan entre ellas las que permiten con mayor fuerza formar profesionales preparados para afrontar ese reto de transformar nuestro sistema educativo en uno más inclusivo:

- Capacidad para comprender la complejidad de los procesos educativos

- Respeto a las diferencias culturales y personales

- Capacidad para preparar, seleccionar o construir materiales didácticos

- Capacidad para promover la calidad de los contextos en los que se desarrolla el proceso educativo

- Capacidad para utilizar la evaluación como elemento regulador y promotor de la mejora

- Capacidad para trabajar en equipo con los compañeros

- Capacidad para dinamizar la construcción participada de reglas de convivencia democrática y afrontar y resolver de forma colaborativa situaciones problemáticas y conflictos interpersonales

- Capacidad para colaborar con los distintos sectores de la comunidad educativa y del entorno

- Diseño y desarrollo de proyectos educativos y unidades de programación que permitan adaptar el curriculum al contexto sociocultural

- Capacidad para promover el aprendizaje autónomo de los alumnos desarrollando estrategias que eviten la exclusión y la discriminación

A estas competencias se deberían añadir aquellas que la Agencia Europea para el Desarrollo de la Educación del Alumnado con Necesidades Educativas Especiales $(2011,2012)$ determina para que los maestros enseñen desde un enfoque inclusivo: valorar y apoyar el progreso de todo el alumnado, trabajar en equipo, emplear diversas metodologías, fomentar experiencias de aprendizaje activas y participativas y diversificar los contenidos y los métodos de evaluación.

$\mathrm{Al}$ analizar en otros estudios (Álvarez Castillo y Buenestado, 2015; López-Torrijo y Mengual-Andrés, 2015; Loraine, 2015; Rodríguez-Gómez et al., 2017; Sharma y Jacobs, 2016) los resultados obtenidos sobre la formación inicial de los futuros profesionales de la educación destaca el hecho de que los estudiantes universitarios, a nivel general, manifiestan actitudes favorables y acordes a los principios y valores que defiende la inclusión. Sin embargo, se observa que dichas actitudes se alteran cuando entran en juego variables de formación y experiencia directa con alumnos con necesidades educativas especiales. De hecho, las principales lagunas formativas se sitúan en la falta de práctica que otorgue a estos estudiantes un mayor dominio de las competencias docentes y un mayor sentimiento de autoeficacia. Es decir, dentro de las tres características que Rouse (2009, citado por Spratta y Florian, 2015) considera que debe reunir un profesor inclusivo. (1) conocer teoría, política y legislación; (2) hacer, convertir el conocimiento en acción; (3) creer en la capacidad de enseñar a todos los niños; podríamos afirmar que la formación inicial del profesorado escasea en las dos últimas.

Si se tiene en cuenta todo lo abordado hasta el momento desde la experiencia como formadores de futuros profesionales de la educación dentro del contexto universitario, se detecta que los estudiantes no están suficientemente preparados para abordar el desafío de la inclusión. Sin embargo, a pesar de la constatación de este hecho y con el fin de poder tomar cartas en el asunto, debe detectarse en qué se está fallando y, por tanto, en qué aspectos habría que fortalecer la formación inicial que se proporciona. Con este fin, el estudio que se presenta tiene como objetivo validar una escala para evaluar la formación en educación inclusiva de estudiantes universitarios de las titulaciones de educación. Para ello se partió de una escala dirigida a profesionales en ejercicio que ha pasado ya una primera validación, el CEFI-R (González-Gil, Martín-Pastor, Orgaz, Poy) y que en estos momentos se encuentra en proceso de revisión editorial para su publicación.

\section{Método}

\section{1- Participantes}

La población objeto de estudio son los profesores que imparten docencia en centros educativos españoles en las distintas etapas en las que se estructura el sistema educativo (Educación Infantil, Educación Primaria, Educación Secundaria y Formación 
Tabla 1. Características de distribución de la muestra de profesionales $(\mathrm{N}=202)$ y estudiantes $(\mathrm{N}=495)$

\begin{tabular}{|c|c|c|}
\hline & \multicolumn{2}{|c|}{ N (Porcentaje) } \\
\hline & Profesionales & Estudiantes \\
\hline \multicolumn{3}{|l|}{ Género } \\
\hline Hombre & $62(30.7)$ & $108(21.8)$ \\
\hline Mujer & $140(69.3)$ & $387(78.2)$ \\
\hline \multicolumn{3}{|l|}{ Edad } \\
\hline Entre 20 y 25 años & & $419(84.6)$ \\
\hline Mayor de 25 años & & $76(15.4)$ \\
\hline Menor de 30 años & $12(6)$ & \\
\hline Entre 30 y 40 años & $58(29)$ & \\
\hline Entre 41 y 50 años & $70(35)$ & \\
\hline Mayor de 50 años & $60(30)$ & \\
\hline \multicolumn{3}{|l|}{ Formación } \\
\hline Maestro en educación infantil & $23(11.4)$ & $175(35.4)$ \\
\hline Maestro en educación primaria & $59(29.2)$ & $203(41.0)$ \\
\hline Maestro en educación especial & $13(6.5)$ & \\
\hline Pedagogía & $15(7.4)$ & $22(4.4)$ \\
\hline Otra titulación (licenciatura: profesores de educación secundaria) & $92(45.5)$ & \\
\hline Máster de Secundaria & & $95(19.2)$ \\
\hline
\end{tabular}

Profesional) y estudiantes de las diferentes ramas de Educación (Maestro en Educación Infantil, Primaria, Pedagogía y el Máster de Secundaria). Se seleccionaron profesores de las diferentes etapas educativas, con distintos años de experiencia y con una formación inicial universitaria diferente y estudiantes de las diferentes ramas de Educación tanto de Grado como de Máster. De este modo, la muestra estuvo formada por un total de 202 profesores (profesionales) y 495 estudiantes. En la tabla 1 se describe la distribución de la misma.

\section{2- Procedimiento}

Para la validación de la escala, se tomó como referencia el cuestionario desarrollado y validado para evaluar la formación docente en inclusión de profesionales de la educación -CEFI-R(González-Gil, Martín-Pastor, Orgaz y Poy) con objeto de validarlo con estudiantes universitarios de último curso de Grado o de Máster de titulaciones de Educación. Lo anterior con el fin de comprobar si dicho cuestionario funcionaba también para evaluar la formación en inclusión de estos estudiantes.

Con el fin de facilitar la recogida de datos, a los profesores participantes en el estudio se les ofrecieron dos posibilidades para contestar al cuestionario: en formato papel, que se hacía llegar a la dirección postal del centro educativo en el que trabajaban o en su versión online a través de un link que se les facilitó por correo electrónico. En lo que respecta a la muestra de estudiantes, los cuestionarios fueron administrados en momentos que los profesores colaboradores que impartían docencia en el último año dejaron disponibles para su cumplimentación. En ambos casos se garantizó la confidencialidad, el anonimato y el uso de la información resultante únicamente con fines investigadores.

\section{Resultados}

Se realizó un análisis de la versión del CEFI-R por separado para la muestra de estudiantes y profesionales. En primer lugar, se analizaron los índices de homogeneidad de los ítems de las diferentes dimensiones, obteniéndose también los estadísticos descriptivos. A continuación, se realizó un Análisis Factorial Exploratorio para analizar la estructura del cuestionario CEFI-R (Bandalos y Finney, 2010; Hoyle y Duvall, 2004). Con posterio- ridad, se obtuvo el grado de consistencia interna, utilizando el alpha de Cronbach por separado para las distintas dimensiones y se calcularon también las intercorrelaciones entre las mismas. Por último, se analizaron las diferencias entre estudiantes y profesionales en cada una de las dimensiones. A continuación, se presentan los resultados obtenidos.

\section{1- Análisis de los ítems de las diferentes dimensiones.}

En la dimensión Concepción de la diversidad, la correlación entre los ítems y la dimensión a la que pertenecían osciló en la muestra de profesionales entre .482 para el ítem 2 “Un niño con necesidades específicas de apoyo educativo interrumpe la rutina del aula y perjudica el aprendizaje de sus compañeros" y .438 para el ítem 1 "Preferiría no tener en mi aula alumnos con necesidades específicas de apoyo educativo"; en la muestra de estudiantes entre .452 para el ítem 2 y .321 para el ítem 3 "Los alumnos con necesidades específicas de apoyo educativo no pueden seguir el día a día del curriculum".

En la dimensión Metodología, en la muestra de profesionales entre .741 para el ítem 6 "Sé cómo elaborar las unidades didácticas y las clases teniendo presente la diversidad de los estudiantes" y .568 para el ítem 9 "Soy capaz de adaptar mis técnicas de comunicación para asegurarme de que todos los alumnos puedan ser incluidos con éxito en el aula ordinaria"; en la muestra de estudiantes entre .775 para el ítem 7 "Sé cómo adaptar mi forma de evaluar a las necesidades individuales de cada uno de mis alumnos" y .478 para el ítem 9.

En la dimensión Apoyos, en la muestra de profesionales entre .713 para el ítem 13 "Considero que el lugar del profesor de apoyo está dentro del aula ordinaria con cada uno de los profesores" y .463 para el ítem 12 "La función del profesor de apoyo es trabajar con todo el alumnado de mi aula"; en la muestra de alumnos entre .639 para el ítem 13 y .351 para el ítem 10 "La planificación conjunta profesor-profesor de apoyo facilitaría que los apoyos se proporcionaran dentro del aula".

En la dimensión Participación en la comunidad, en la muestra de profesionales entre .608 para el ítem 15 "Es fundamental que haya una relación muy estrecha entre el profesorado y el resto de agentes educativos" y .418 para el ítem 14 "El proyecto educativo debería revisarse con la participación de los diferentes agentes 
Tabla 2. Índices de homogeneidad corregidos de los ítems

\begin{tabular}{|c|c|c|c|c|}
\hline \multirow[b]{2}{*}{ Indicadores e ítems } & \multicolumn{2}{|c|}{ Profesionales } & \multicolumn{2}{|c|}{ Estudiantes } \\
\hline & $\mathrm{M}(\mathrm{SD})$ & $\mathrm{R}_{\mathrm{i}(\mathrm{x}-\mathrm{i})}$ & $\mathrm{M}(\mathrm{SD})$ & $\mathrm{R}_{\mathrm{i}(\mathrm{x}-\mathrm{i})}$ \\
\hline \multicolumn{5}{|l|}{ Concepción de la Diversidad } \\
\hline 1. Preferiría no tener en mi aula alumnos con necesidades específicas de apoyo educativo & $\begin{array}{c}2.97 \\
(1.09)\end{array}$ & .438 & $\begin{array}{l}3.38 \\
(0.82)\end{array}$ & .416 \\
\hline $\begin{array}{l}\text { 2. Un niño con necesidades específicas de apoyo educativo interrumpe la rutina del aula y perjudica el } \\
\text { aprendizaje de sus compañeros }\end{array}$ & $\begin{array}{c}2.92 \\
(0.87)\end{array}$ & .482 & $\begin{array}{l}3.37 \\
(0.73)\end{array}$ & .452 \\
\hline $\begin{array}{l}\text { 3. Los alumnos con necesidades específicas de apoyo educativo no pueden seguir el día a día del } \\
\text { curriculum }\end{array}$ & $\begin{array}{c}2.41 \\
(0.89)\end{array}$ & .451 & $\begin{array}{l}3.06 \\
(0.80)\end{array}$ & .321 \\
\hline $\begin{array}{l}\text { 4.Me preocupa que mi carga de trabajo se incremente si tengo alumnos con necesidades específicas de } \\
\text { apoyo educativo en mi clase }\end{array}$ & $\begin{array}{c}2.54 \\
(1.06)\end{array}$ & .461 & $\begin{array}{c}3.22 \\
(0.91)\end{array}$ & .357 \\
\hline \multicolumn{5}{|l|}{ Metodología } \\
\hline $\begin{array}{l}\text { 5. Sé cómo enseñar a cada uno de mis alumnos de manera diferente en función de sus características } \\
\text { individuales }\end{array}$ & $\begin{array}{c}2.77 \\
(0.85)\end{array}$ & .639 & $\begin{array}{l}2.66 \\
(0.86)\end{array}$ & .692 \\
\hline $\begin{array}{l}\text { 6. Sé cómo elaborar las unidades didácticas y las clases teniendo presente la diversidad de los estudi- } \\
\text { antes }\end{array}$ & $\begin{array}{l}3.10 \\
(0.78)\end{array}$ & .741 & $\begin{array}{l}2.75 \\
(0.84)\end{array}$ & .747 \\
\hline 7. Sé cómo adaptar mi forma de evaluar a las necesidades individuales de cada uno de mis alumnos & $\begin{array}{l}3.13 \\
(0.72)\end{array}$ & .733 & $\begin{array}{c}2.83 \\
(0.81)\end{array}$ & .775 \\
\hline $\begin{array}{l}\text { 8. Sé cómo manejar y adaptar los materiales didácticos para responder a las necesidades de cada uno } \\
\text { de mis alumnos }\end{array}$ & $\begin{array}{l}3.00 \\
(0.72)\end{array}$ & .719 & $\begin{array}{c}2.82 \\
(0.74)\end{array}$ & .765 \\
\hline $\begin{array}{l}\text { 9. Soy capaz de adaptar mis técnicas de comunicación para asegurarme de que todos los alumnos } \\
\text { puedan ser incluídos con éxito en el aula ordinaria }\end{array}$ & $\begin{array}{c}3.18 \\
(0.68)\end{array}$ & .568 & $\begin{array}{c}3.07 \\
(0.72)\end{array}$ & .478 \\
\hline
\end{tabular}

Apoyos

10. La planificación conjunta profesor-profesor de apoyo facilitaría que los apoyos se proporcionaran dentro del aula

3.52

$(0.72)$

11. Creo que la mejor manera de proporcionar apoyo a los alumnos es que el profesor de apoyo se incorpore al aula en lugar de hacerlo en el aula de apoyo

12. La función del profesor de apoyo es trabajar con todo el alumnado de mi aula

13. Considero que el lugar del profesor de apoyo está dentro del aula ordinaria con cada uno de los profesores

Participación de la Comunidad

14. El proyecto educativo debería revisarse con la participación de los distintos agentes de la comunidad educativa (profesores, padres, alumnos, ...)

15. Es fundamental que haya una relación estrecha entre el profesorado y el resto de agentes educativos (AMPA, asociación de vecinos, consejo escolar, ...)

\begin{tabular}{cccc}
3.22 & .418 & 3.59 & .543 \\
$(0.88)$ & & $(0.66)$ & \\
3.45 & .608 & 3.71 & .692 \\
$(0.79)$ & & $(0.59)$ & \\
3.46 & .490 & 3.71 & .596 \\
$(0.68)$ & & $(0.54)$ & \\
\hline
\end{tabular}

16. El centro debe trabajar de forma conjunta con los recursos del barrio (biblioteca, servicios sociales, servicios sanitarios, ...)

de la comunidad educativa"; en el caso de los estudiantes entre .692 también para el ítem 15 y .543 para el ítem 14 .

En la tabla 2 se recogen las puntuaciones para cada ítem.

Se obtienen unas buenas correlaciones entre los ítems y la dimensión a la que corresponden, tanto en la muestra de profesionales, que en todos los casos se superan las correlaciones de .40 , como en la de los estudiantes, donde las correlaciones son superiores a .30. En la dimensión que se observan los mejores resultados, en ambos colectivos, es en la de Metodología.

\section{2- Análisis de la estructura del cuestionario.}

En el análisis previo al Análisis Factorial Exploratorio, se obtuvo que el valor de Kaiser-Meyer-Olkin excedía el valor recomentado de .60 tanto en la muestra de profesionales (.78) como en la de estudiantes (.79); también se obtuvieron resultados significativos en ambos casos en la Prueba de Esfericidad de Bartlett $\left(\mathrm{c}^{2}=2536.17(\mathrm{df}=120), \mathrm{p}<.001\right.$, en el caso de los estudiantes $\mathrm{y}$ $c^{2}=1069.29(\mathrm{df}=120), \mathrm{p}<.001$, en el de los profesionales).
Se realizó una Factorización de Ejes Principales para comprobar el grado de ajuste de los ítems a la estructura del CEFI-R en las dos muestras. Como se esperaba que las dimensiones estuvieran intercorrelacionadas, se utilizó una rotación oblicua. La extracción de los factores se realizó en función de los autovalores y el gráfico de sedimentación, fueron extraídos aquellas dimensiones con autovalores superiores a uno.

En ambas muestras fueron extraídos cuatro factores (correspondienteas a las cuatro dimensiones) que explicaban el $61.4 \%$ de la varianza en el caso de los profesionales y el 59.6\%, en el caso de los estudiantes. Se presenta las soluciones rotadas para las dos muestras en las tablas 3 y 4 . Como se puede observar, en ambos casos, el primer factor es Metodología y el tercero es Concepción de la Diversidad. Los factores Apoyos y Participación en la Comunidad intercambian el orden: en el caso de los profesionales, Apoyos es el segundo y Participación, el cuarto y en el caso de los estudiantes al contrario.

En cuanto a los ítems incluidos en los factores (dimensiones), son los mismos en Metodología y Concepción de la Diversidad para 
ambos grupos. Pero en el caso de Apoyos y Participación de la Comunidad los estudiantes ponderaban en los dos factores e incluso el ítem 10, que corresponde a la dimension de Apoyos, contribuía más en Participación de la Comunidad.

\section{3- Análisis de la consistencia interna}

La dimensión que mostró mejores índices de fiabilidad para ambas muestras fue la de Metodología. Los índices de fiabilidad también fueron buenos para las dos muestras en el caso de la

Tabla 3. Factores y cargas factoriales de los ítems en la muestra de profesionales

\begin{tabular}{|c|c|c|c|c|}
\hline Ítems & $\begin{array}{l}\text { Factor 1: } \\
\text { Dimensión } \\
\text { Metodología }\end{array}$ & $\begin{array}{l}\text { Factor 2: } \\
\text { Dimensión } \\
\text { Apoyos }\end{array}$ & $\begin{array}{l}\text { Factor 3: } \\
\text { Dimensión } \\
\text { Concepción } \\
\text { Diversidad }\end{array}$ & $\begin{array}{c}\text { Factor 4: } \\
\text { Dimensión } \\
\text { Participación } \\
\text { Comunidad }\end{array}$ \\
\hline Ítem 7 & .816 & & & \\
\hline Ítem 6 & .805 & & & \\
\hline Ítem 8 & .801 & & & \\
\hline Ítem 5 & .695 & & & \\
\hline Ítem 9 & .624 & & & \\
\hline Ítem 13 & & .857 & & \\
\hline Ítem 11 & & .806 & & \\
\hline Ítem 10 & & .588 & & \\
\hline Ítem 12 & & .527 & & \\
\hline Ítem 2 & & & .620 & \\
\hline Ítem 3 & & & .595 & \\
\hline Ítem 4 & & & .587 & \\
\hline Ítem 1 & & & .566 & \\
\hline Ítem 15 & & & & .860 \\
\hline Ítem 16 & & & & .642 \\
\hline Ïtem14 & & & & .498 \\
\hline$\%$ varianza explicada & 25.21 & 16.96 & 11.56 & 8.63 \\
\hline
\end{tabular}

Tabla 4. Factores y cargas factoriales de los ítems en la muestra de estudiantes.

\begin{tabular}{|c|c|c|c|c|}
\hline Ítems & $\begin{array}{c}\text { Factor 1: } \\
\text { Dimensión } \\
\text { Metodología }\end{array}$ & $\begin{array}{c}\text { Factor 2: } \\
\text { Dimensión } \\
\text { Participación } \\
\text { Comunidad }\end{array}$ & $\begin{array}{l}\text { Factor 3: } \\
\text { Dimensión } \\
\text { Concepción } \\
\text { Diversidad }\end{array}$ & $\begin{array}{c}\text { Factor 4: } \\
\text { Dimensión } \\
\text { Apoyos }\end{array}$ \\
\hline Ítem 7 & .852 & & & \\
\hline Ítem 8 & .824 & & & \\
\hline Ítem 6 & .816 & & & \\
\hline Ítem 5 & .744 & & & \\
\hline Ítem 9 & .503 & & & \\
\hline Ítem 15 & & .859 & & .320 \\
\hline Ítem 16 & & .699 & & .346 \\
\hline Ítem 14 & & .612 & & .345 \\
\hline Ítem 10 & & .497 & & .444 \\
\hline Ítem 2 & & & .653 & \\
\hline Ítem 1 & & & .614 & \\
\hline Ítem 4 & & & .454 & \\
\hline Ítem 3 & & & .417 & \\
\hline Ítem 11 & & .433 & & .843 \\
\hline Ítem 13 & & .435 & & .798 \\
\hline Ítem12 & & & & .397 \\
\hline$\%$ varianza explicada & 25.08 & 16.48 & 10.46 & 7.58 \\
\hline
\end{tabular}


Tabla 5. Intercorrelaciones entre las dimensiones del CEFI-R y fiabilidad para cada dimensión

\begin{tabular}{|c|c|c|c|c|c|c|}
\hline Dimensiones & 1 & 2 & 3 & 4 & $\begin{array}{l}\text { a de Cronbach } \\
\text { Profesionales }\end{array}$ & $\begin{array}{l}\text { a de Cronbach } \\
\text { Estudiantes }\end{array}$ \\
\hline $\begin{array}{l}\text { 1. Concepción de la diversidad } \\
\text { (4 ítems) }\end{array}$ & & $.21^{* *}$ & $.24^{* *}$ & .09 & .67 & .60 \\
\hline $\begin{array}{l}\text { 2. Metodología } \\
\text { (5 ítems) }\end{array}$ & $.20^{* * *}$ & & $.15^{*}$ & .07 & .86 & .87 \\
\hline $\begin{array}{l}\text { 3.Apoyos } \\
\text { (4 ítems) }\end{array}$ & $.12^{* *}$ & $.16^{* *}$ & & $.36^{* * *}$ & .78 & .68 \\
\hline $\begin{array}{l}\text { 4.Participación de la Comunidad } \\
\text { (3 ítems) }\end{array}$ & $.19^{* * *}$ & $.16^{* *}$ & $.45^{* * *}$ & & .68 & .77 \\
\hline
\end{tabular}

${ }^{*} \mathrm{p}<.05,{ }^{* *} \mathrm{p}<.01,{ }^{* * *} \mathrm{p}<.001$

Por encima de la diagonal principal se incluyen las intercorrelaciones para la muestra de profesionales y por debajo para la de estudiantes.

dimensión de Apoyos, por encima de .70 en los profesionales y alrededor de .70 en la de estudiantes. En el caso de Participación de la Comunidad, ocurre lo contrario, la fiabilidad estimada en el caso de los estudiantes fue superior a .70 y la de los profesionales estaba en torno a .70. Por último, la dimensión en la que se obtuvieron índices más bajos fue en Concepción de la diversidad con un valor próxima a .70 en los profesionales y de .60 en los estudiantes (Ver Tabla 5).

Como se esperaba, se encontraron intercorrelaciones entre las dimensiones. Las correlaciones más elevadas se encontraron, en ambas muestras, entre las dimensiones de Apoyos y Participación de la Comunidad. Las correlaciones entre Concepción de la Diversidad y Participación de la Comunidad y entre Metodología y Participación de la Comunidad fueron significativas en el caso de los estudiantes pero no, en el de los profesionales (ver tabla 5).

\subsection{Diferencias en las dimensiones entre profesionales y estudiantes}

Se encontraron diferencias significativas entre dimensiones $\left[\mathrm{F}(1,2085)=146.18, \mathrm{p}<.001\right.$, eta $\left.{ }^{2}=.17\right]$; realizados los contrastes a posteriori con el ajuste de Bonferroni, se encontraron diferencias significativas $(\mathrm{p}<.001)$ entre la dimensión de Participación y todas las demás dimensiones: la puntuaciones eran significativamente más altas en esta dimensión $(\mathrm{M}=3.58, \mathrm{DT}=0.56)$ que en el resto [Apoyos $(M=3.16, D T=0.65)$, Metodología $(M=2.89$, $\mathrm{DT}=0.64)$ y Concepción de la Diversidad $(\mathrm{M}=2.98$, DT=0.74)]. La diferencia entre las dimensiones de Metodología y Apoyos también era significativa $(p=.001)$ : la media en apoyos era significativamente más elevada.

También encontramos diferencias significativas $\left[\mathrm{F}(1,695)=73.41, \mathrm{p}<.001\right.$, eta $\left.^{2}=.10\right]$, entre los Profesionales $(\mathrm{M}=2.88$, $\mathrm{DT}=0.33)$ y los Estudiantes $(\mathrm{M}=3.26$, $\mathrm{DT}=0.36)$ : las puntuaciones de los Estudiantes eran significativamente más altas que las de los profesionales.

Tabla 6. Descriptivos en las dimensiones del CEFI-R en profesionales y estudiantes

\begin{tabular}{lccc}
\hline & $\begin{array}{c}\text { Profesionales } \\
(\mathrm{n}=202) \\
\mathbf{M}(\mathrm{SD})\end{array}$ & $\begin{array}{c}\text { Estudiantes } \\
(\mathrm{n}=495) \\
\mathbf{M}(\mathrm{SD})\end{array}$ & $\begin{array}{c}\mathrm{F}(\mathrm{gl}) \\
\mathrm{p}, \eta^{2}\end{array}$ \\
\hline 1. Concepción de la diversidad & $2.71(0.70)$ & $3.26(0.56)$ & $\begin{array}{r}\mathrm{F}(1,695)=120.04 \\
\mathrm{p}<.001 \eta^{2}=.15\end{array}$ \\
2. Metodología & $3.04(0.63)$ & $2.83(0.65)$ & $\begin{array}{r}\mathrm{F}(1,695)=15.81 \\
\mathrm{p}<.001 \eta^{2}=.02 \\
\mathrm{~F}(1,695)=77.44 \\
\mathrm{p}<.001 \eta^{2}=.10\end{array}$ \\
3.Apoyos & $2.84(0.72)$ & $3.29(0.57)$ & $\begin{array}{r}\mathrm{F}(1,695)=41.70 \\
\mathrm{p}<.001 \eta^{2}=.06\end{array}$ \\
\hline
\end{tabular}

Por último, la interacción profesionales/estudiantes por dimensión también fue significativa $[\mathrm{F}(3,2085)=57.10, \mathrm{p}<.001$, eta $\left.^{2}=.08\right]$; realizados los contrastes a posteriori, se obtuvieron diferencias significativas en todas las dimensiones: en todos los casos a excepción de Metodología, los estudiantes manifestaban actitudes más positivas que los profesionales; en el caso de la dimensión Metodología eran los profesionales los que mostraban actitudes más positivas.

Figura 1. Puntuaciones en las diferentes dimensiones del CEFI-R de profesionales y estudiantes

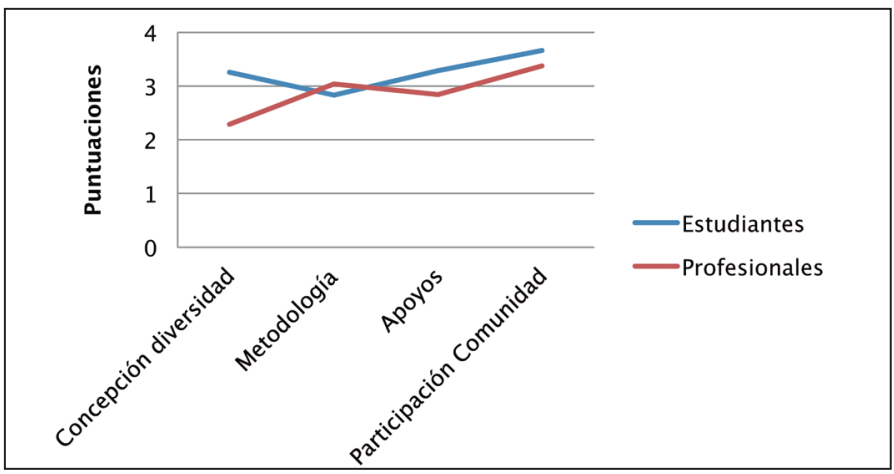




\section{Discusión y Conclusiones}

El objetivo que dio inicio a este trabajo era la validación de una escala que permitiese evaluar la formación que los futuros maestros tienen para trabajar en entornos educativos inclusivos. Se puede afirmar que se ha conseguido en la medida en que se dispone de un instrumento válido y fiable para realizar esa evaluación. Los resultados obtenidos muestran un instrumento multidimensional, compuesto por 16 ítems que miden las actitudes, competencias, habilidades y destrezas docentes para este colectivo, en base a cuatro dimensiones:

1. Concepción de la diversidad. Esta dimensión recoge la valoración personal sobre el concepto de diversidad, el lugar y la forma en que se contempla la escolarización de los alumnos, la política educativa en la que se sustentan estas decisiones y, en definitiva, la interpretación individual de la educación inclusiva

2. Metodología. Contempla la perspectiva desde la que se define cada uno de los elementos que forman parte del currículum (estrategias metodológicas, recursos, materiales, técnicas de comunicación, evaluación) y si los estudiantes se consideran preparados para realizarlo desde la óptica de la inclusión

3. Apoyos. Aborda el concepto de apoyo, el rol que debe desempeñar el profesor de apoyo, los potenciales destinatarios de dicho apoyo y el lugar donde se proporciona $y$, por tanto, el trabajo colaborativo entre este profesional y el resto de profesores

4. Participación de la comunidad. Recoge información sobre el trabajo colaborativo entre el centro educativo y el resto de agentes de la comunidad, así como del uso de los recursos que, más allá del recinto de la escuela, nos ofrece el entorno

Se han obtenido buenos índices de fiabilidad para ambas muestras en todas las dimensiones presentadas, destacando en los dos casos la de metodología por su elevada fiabilidad y la de concepción de la diversidad con la fiabilidad más baja, aunque en todo caso, aceptable.

$\mathrm{Al}$ analizar las puntuaciones obtenidas en cada dimensión y compararlas con las de los profesores en ejercicio se puede observar que las medias obtenidas por los primeros son superiores a las de los docentes en tres de las cuatro dimensiones (concepción de la diversidad, apoyos y participación de la comunidad), lo que pone de manifiesto que, si bien los estudiantes presentan mejores actitudes hacia la diversidad y un concepto de inclusión más cercano a la definición de la misma que los profesores, se sienten menos competentes a nivel metodológico para trabajar desde una perspectiva inclusiva y para proporcionar a sus alumnos la respuesta que requiere las necesidades que presentan. Así se ha reflejado en las puntuaciones en metodología, única dimensión en que los profesionales han obtenido valores superiores. Lo anterior implica, de acuerdo con la información recopilada a través de los ítems que definen este aspecto, carencias formativas en la práctica educativa diaria: cómo elaborar unidades didácticas, como adaptar los materiales didácticos y la forma de evaluar o el uso de técnicas de comunicación diversas. Todo ello concuerda con los resultados obtenidos recientemente en los trabajos de Álvarez y Buenestado (2015), López-Torrijo y Mengual-Andrés (2015), Loraine (2015), Rodríguez-Gómez el al. (2017), Sharma y Jacobs (2016), entre otros.

No obstante, no se puede perder de vista la importancia que, tal y como reflejan Álvarez y Buenestado (2015), Kitsantas (2012) y Mosia (2014), esas actitudes favorables tienen para la adquisición de herramientas y estrategias que puedan compensar las carencias formativas detectadas en este trabajo, lo que permite ser positivos y confiar en las oportunidades que la formación universitaria puede proporcionar al respecto. Formación que, indudablemente, se debe replantear tomando como referencia las conclusiones obtenidas en las Jornadas de Universidad y Educación Inclusiva celebradas en Oviedo en 2017. Así, la formación en inclusión se debe realizar de manera transversal y holística en cuanto a metodología, contenidos, discursos y experiencias que muestren a los futuros maestros que el cambio es posible. De esta manera lo reflejan también Florian et al. (2010) al defender que la preparación de los futuros maestros necesariamente debe transmitir un enfoque de enseñanza-aprendizaje para todos los alumnos, dejando al margen designaciones como "la mayoría" o "algunos", apostando por la innovación didáctica que promueva nuevos espacios sociales inclusivos.

Los resultados también desvelan cómo los estudiantes tienen una concepción errónea del apoyo, una concepción más vinculada a un planteamiento integrador de la educación: ayuda que el profesor especialista presta de manera individual, fuera del aula ordinaria y al margen del currículum que en la misma se trabaja, a aquellos alumnos con necesidades educativas diagnosticadas. Esta visión concuerda con lo que plantea Parrilla (2007) al afirmar que el apoyo es entendido en muchas ocasiones como una delegación, una separación del diferente. En contraposición, la inclusión defiende un modelo de apoyo completamente distinto, en el que se abandona su matiz experto y prescriptivo y lo enmarca dentro de todas las actividades que un centro educativo realiza con el fin de aumentar su capacidad para responder a la diversidad del alumnado (Booth y Ainscow, 2011; Echeita, 2013). Así, el apoyo también se genera cuando los profesores planifican juntos sus lecciones teniendo en cuenta a todos sus estudiantes o cuando los propios alumnos se ayudan unos a otros.

En relación con esta última idea, las puntuaciones obtenidas por los estudiantes en las dimensiones de apoyos y participación de la comunidad, las altas correlaciones entre ambas dimensiones y el hecho de que los ítems que las definen ponderen en los dos factores que dan lugar a las mismas pone de manifiesto además que los futuros maestros no tienen claro cuál puede ser el papel de otros profesionales dentro del aula, acompañando al docente responsable de la asignatura ni que la enseñanza y el trabajo en un centro educativo debe ser una tarea compartida con el resto de agentes de la comunidad. Un ejemplo que ilustra este hecho es que el ítem 10, de contenido claramente relacionado con la dimensión de apoyos, tenga un peso superior en la de participación de la comunidad. Este aspecto muestra la imagen que aún persiste de un profesorado que continúa trabajando desde un enfoque individualista, alejado de valores como compartir (estrategias, recursos, inquietudes) con otros compañeros, como herramienta fundamental de apoyo a la labor docente (Booth y Ainscow, 2011). Todo ello revela la necesidad de seguir indagando en el contenido de los ítems que definen ambas dimensiones y su influencia en la composición de la escala, para depurarla al máximo y ajustarla a los destinatarios de la misma, mientras se modifican los programas de formación de los futuros profesionales de la educación, incorporando todo lo que se deriva de los resultados presentados.

En base a todo lo expuesto la universidad, como institución responsable de la preparación de estos profesionales, debe estar inmersa en un proceso de mejora continua de la formación de sus estudiantes a partir de un modelo de educación, de escuela que, lejos de anclarse en la uniformidad, despliegue una gran variedad de experiencias en las que los alumnos encuentren opor- 
tunidades para aprender satisfactoriamente. Todo ello permitirá, como efecto colateral, mejorar la calidad del sistema educativo y de sus resultados y dar pasos firmes en su transformación hacia un sistema más inclusivo.

\section{Referencias Bibliográficas}

Acedo, C. (2011). Preparing teachers for inclusive education. Prospects, 41, 301-202

Agencia Europea para el Desarrollo de la Educación del Alumnado con Necesidades Educativas Especiales (2011). Formación del profesorado para la educación inclusiva en Europa. Retos y oportunidades. Odense, Dinamarca: European Agency for Development in Special Needs Education

Agencia Europea para el Desarrollo de la Educación del Alumnado con Necesidades Educativas Especiales (2012). Formación del profesorado para la educación inclusiva. Perfil profesional del docente en la educación inclusiva. Odense, Dinamarca: European Agency for Development in Special Needs Education

Álvarez Castillo, J.L. y Buenestado Fernández, M. (2015). Predictores de las actitudes hacia la inclusión de alumnado con necesidades educativas especiales en futuros profesionales de la educación. Revista Complutense de Educación, 26 (3), 627-645. doi: http://dx.doi.org/10.5209/rev_RCED.2015.v26.n3.44551

ANECA (2015). Libro Blanco de Grado en Magisterio, vol.1. Madrid: Autor

Arnáiz, P. y Guirao, J.M. (2015). La autoevaluación de centros en España para la atención a la diversidad desde una perspectiva inclusiva: ACADI. Revista Electrónica Interuniversitaria de Formación del Profesorado, 18 (1), 45-101

Avramidis, E. \& Kalyra, E. (2007). The influence of teaching experience and professional development on greek teachers 'attitudes towards inclusion. European Journal of Special Needs Education, 22(4), 367-389. doi: 10.1080/08856250701649989

Bandalos, D. L. y Finney, S. J. (2010). Factor analysis: Exploratory and confirmatory. In G. R. Hancock \& R. O. Mueller (Eds.), The reviewer's guide to quantitative methods in the social sciences (pp. 93-114). New York, NY: Routledge.

Booth, T. \& Ainscow, M. (2011). Index for Inclusión. Developing learning and participation in schools ( $3^{a}$ ed.). Manchester: CSIE

Bricall, J.M. (2000). Informe Universidad 2000. Barcelona: Conferencia de Rectores

Colmenero, M. J., Pantoja, A. \& Pegajalar, M.C. (2015). Percepciones sobre atención a la diversidad en la formación inicial del profesorado en Educación Secundaria. Revista Complutense de Educación, 26(1), 101-120

Dias, P.C. \& Cadime, I. (2016). Effects of personal and professional factor son teachers' attitudes towards inclusion in preschool. European Journal of Special Needs Education, 31(1), 111123. doi: 10.1080/08856257.2015.1108040

Donnelly, V. \& Watkins, A. (2011). Teacher education for inclusion in Europe. Prospects, 41, 341-353. doi: 10.1007/s11125011-9199-1

Echeita, G. (2013). Inclusión y exclusión educativa. De Nuevo "Voz y quebranto". Revista Iberoamericana sobre Calidad, Eficacia y Cambio en Educación, 11(2), 99-118

Escudero, J.M. y Martínez, B. (2011). Educación inclusiva y cambio escolar. Revista Iberoamericana de Educación, 55, 85-105

Florian, L., Young, K. y Rouse, M. (2010). Preparing teachers for inclusive and diverse educational environments: studying curricular reform in an initial teacher education course. International Journal of Inclusive Education, 14 (7), 709-722.doi: 10.1080/13603111003778536

Forteza, D. (2011). Algunas claves para repensar la formación del profesorado sobre la base de la inclusión. Revista Interuniversitaria de Formación del Profesorado, 70(25,1), 127-144

Hoyle, R. H. y Duvall, J. L. (2004). Determining the number of factors in exploratory and confirmatory factor analysis. In D. Kaplan (Ed.), The SAGE handbook of quantitative methodology for social sciences (pp. 301-315). Thousand Oaks, CA: Sage.

Kitsantas, A. (2012). Teacher efficacy scale for classroom diversity (TESCD): A validation study. Profesorado. Revista de curriculum y formación del profesorado, 16 (1), 35-44

Kraska, J. \& Boyle, C. (2014). Attitudes of Preschool and Primary School Pre-service Teachers towards Inclusive Education. Asia-Pacific Journal of Teacher Education 42(3), 228-246. doi:10. 1080/1359866X.2014.926307

López-Torrijo, M. y Mengual-Andrés, S. (2015). An attack on inclusive education in secondary education. Limitations in initial teacher training in Spain. New approaches in educational research, 4 (1), 9-17. doi: https://doi.org/10.7821/naer.2015.1.100

Loreman, T., McGhie-Richmond, D., Kolupayeva, A., Taranchenko, O., Mazin, D., Crocker, C. y Petryshyn, R. (2016). A Canada-Ukraine collaborative initiative for introducing inclusive education for children with disabilities in Ukraine: participant perspectives. School Effectiveness and School Improvement, 27(1), 24-44

Loraine, Mc. (2015). Beginning teachers and inclusive education: frustrations dilemas and growth. International Journal of Inclusive Education, 20 (4), 383-396. doi: http://dx.doi.org/10.1080/1 $\underline{3603116.2015 .1081635}$

Molina, S. \& Holland, CH. (2010). Educación especial e inclusión: aportaciones desde la investigación. Revista Educación y Pedagogía, 22(56), 31-43.

Mosia, P.A. (2014) Threats to inclusive education in Lesotho: An overview of policy and implementation challenges. Africa Education Review, 11 (3), 292-310.

Muntaner, J.J., Rosselló, M.R. y De la Iglesia, B. (2016). Buenas prácticas en educación inclusiva. Educatio Siglo XXI, 34 (1), 31-50

Parrilla, A. (2007). Inclusive Education in Spain: a view from inside. En Barton, L. y Amstrong, F. (Eds.). Policy, Experience and Change: Cross Cultural Reflections on Inclusive Education (pp. 19-36). Dordrecht: Springer.

Pujolás, P. (2015). Marques de foc. Lliçons de pedagogía. Vic: Servei de Publicacions de la UVic-UCC

Reoyo, N., Carbonero, M. Á. y Martín, L.J. (2017). Características de eficacia docente desde las perspectivas del profesorado y futuro profesorado de secundaria. Revista de Educación, 376, 62-86. doi: 10.4438/1988-592X-RE-2017-376-344

Robinson, D. (2017). Effective inclusive teacher education for special educational needs and disabilities: Some more thoughts on the way forward. Teaching and Teacher Education, 61, 164-178. doi: https://doi.org/10.1016/j.tate.2016.09.007

Rodríguez-Gómez, D., Armengol, C. y Meneses, J, (2017). La adquisición de las competencias profesionales a través de las prácticas curriculares de la formación inicial de maestros. Revista de Educación, 376, 229-251. doi: 10.4438/1988-592X-RE2017-376-350

Sharma, U. \& Jacobs, DT. K. (2016). Predicting in-service educators' intentions to teach in inclusive classrooms in India and Australia. Teaching and Teacher Education, 55, 13-23. doi:10.1016/j.tate.2015.12.004

Skilar, C. (2013). El lugar del otro en los discursos sobre la inclusion y la diversidad. Buenos Aires: EUA

Spratta, J. y Florian, L. (2015). Inclusive pedagogy: from learning to action. Supporting each individual in the context of "everybody". Teaching and Teacher Education, 49, 89-96. doi: https://doi.org/10.1016/j.tate.2015.03.006

UNESCO (2017). La educación transforma vidas. Paris: Autor

UNESCO (2016). A Review of Evaluative Evidence on Teacher Policy. París: Unesco. Recuperado de http://unesdoc.unesco.org/ images/0024/002443/244373e.pdf

UNESCO (2015). Replantear la educación ¿Hacia un bien común mundial? Paris: UNESCO 